\title{
Oblique particle-wall collisions in a liquid
}

\author{
By G. G. JOSEPH AND M. L. HUNT \\ Division of Engineering and Applied Sciences, California Institute of Technology, \\ Pasadena, CA 91125, USA
}

(Received 16 September 2003 and in revised form 10 February 2004)

This paper presents experimental measurements of the approach and rebound of a particle colliding obliquely with a wall in a viscous fluid. Steel and glass particles $12.7 \mathrm{~mm}$ in diameter were used. The experiments were performed using a thick Zerodur wall (a glass-like material) with various mixtures of glycerol and water. Normal and tangential coefficients of restitution were defined from the ratios of the respective velocity components at the point of contact just prior to and after impact. These coefficients account for losses due to lubrication effects and inelasticity. A third parameter, a coefficient of sliding friction, provides a measure of the tangential force acting on the particle as it slides during a collision.

Oblique collisions in a fluid are qualitatively similar to oblique collisions in a dry system, with a lowered friction coefficient dependent on surface roughness. For smooth surfaces the friction coefficient is drastically reduced due to lubrication effects. A theoretical model that takes into account the dependence of viscosity on pressure is proposed to explain the observed tangential force acting on a smooth sphere during an oblique collision. The model relies on an inferred uniform temperature increase within the lubrication layer, a consequence of viscous heating during impact. The tangential force felt by the particle is expressed as a friction coefficient dependent on the viscosity within the lubrication layer. The viscosity increases owing to pressure effects and decreases owing to thermal effects. For rough surfaces the friction coefficient is comparable to that measured in dry systems, since the surface asperities may interact with each other through the lubrication layer.

\section{Introduction}

The mechanisms of impact and rebound of solid particles in a multi-phase system are of interest over a wide range of applications, including mixing, slurry transport, particle deposition and erosion. Any modelling of these flows requires a detailed understanding of the mechanics of individual collisions. In dry granular flows where the effect of the interstitial fluid is negligible, the energy dissipation due to the inelasticity of the contacts (plasticity, vibrations, cohesivity etc.) is often characterized by a coefficient of restitution $e_{d r y}$, defined by the ratio of the rebound velocity to the impact velocity. In an elastic collision $e_{d r y}=1$, whereas for a perfectly plastic collision $e_{d r y}=0$; collisions with inelastic losses lie in between.

In a similar manner, an effective coefficient of restitution $e$ may be useful in describing a collision in which the effects of the interstitial fluid are important. The knowledge of an effective coefficient of restitution that accounts for the combined effects of the interstitial fluid (added mass, viscous dissipation, compressibility etc.) and the inelasticity of the contact would be useful for numerical simulations of liquidsolid flows (Hu 1996; Glowinski et al. 1999; ten Cate et al. 2002; Nguyen \& Ladd 
2002). In these simulations, the motion of the interstitial fluid is calculated directly; however, the solid surfaces are not allowed to touch because contact would break the lattice modelling of the fluid. When two solid particles come within one grid spacing, fluid nodes are excluded from regions between the solid surfaces, leading to a loss of mass conservation. A repulsive force between the particles is incorporated to prevent contact between solid surfaces. Potapov, Hunt \& Campbell (2001) proposed a combination of smoothed particle hydrodynamics (SPH) and the discrete element method to model flows containing macroscopic solid particles in a viscous liquid. Their SPH model breaks down when the solid particle separation becomes smaller than a kernel width, since a mono-layer of smoothed fluid particles becomes trapped between the solid surfaces. A model based on an effective coefficient of restitution could be used to approximate the solid contacts in liquid-solid flow simulations, significantly improving computational efficiency.

Brenner (1961) analysed the problem of a rigid sphere of density $\rho_{p}$ and radius $R$ normally moving toward a wall at small Reynolds number $\left(R e=2 \rho_{f} R V / \mu\right.$, where $V$ is the approach velocity; $\rho_{f}$ and $\mu$ are the fluid density and dynamic viscosity, respectively). His results show that the hydrodynamic force diverges as the gap separation $h$ tends to zero, preventing any rebound from occurring. Christensen (1970) and Davis, Serayssol \& Hinch (1986) allowed the particle surfaces to deform elastically owing to the increase in hydrodynamic pressure, storing some of the incoming kinetic energy as elastic strain energy. For these elastohydrodynamic (EHD) conditions, Christensen (1970) concluded that the relative approach velocity of two spheres varies according to two relatively independent mechanisms: for gap separations larger than a transition film thickness, the motion is dictated by viscous effects; for smaller gap separations, the motion depends upon elastic deformation. The EHD analysis of Davis et al. (1986) showed that the rebound of the particle after collision depends on the particle Stokes number $S t=M v_{0} / 6 \pi \mu R^{2}=\operatorname{Re}\left(\rho_{p} / \rho_{f}\right) / 9$ and an elasticity parameter $\epsilon=4 \mu v_{0} R^{3 / 2} / \pi E^{*} x_{0}^{5 / 2}$, where $x_{0}$ is the position within the gap between the undeformed surfaces at which the velocity is $v_{0}$. The reduced modulus $E^{*}=\left[\left(1-v_{1}^{2}\right) / E_{1}+\left(1-v_{2}^{2}\right) / E_{2}\right]^{-1}$ is a function of the Young's moduli $E_{1}, E_{2}$ and Poisson's ratios $v_{1}, v_{2}$ of the two bodies. For $S t<S t_{c}$, where $S t_{c}$ is a critical Stokes number, Davis et al. (1986) predicted that no rebound occurs.

During an EHD collision, fluid always remains in the gap between the particles and physical contact between surfaces does not occur. For near-normal EHD collisions at $S t>S t_{c}$, Davis et al. (1986) predicted the minimum gap separation $h_{m}$ to be approximately $1 / 4$ to $1 / 3$ of an elastic lengthscale $x_{1}=\left(4 \mu v_{0} R^{3 / 2} / \pi E^{*}\right)^{2 / 5}$ inspired by the elasticity parameter $\epsilon$. Their result shows that $h_{m}$ increases with increasing normal impact velocity. Smart \& Leighton (1989) and Joseph et al. (2001) argued that, since $h_{m}$ is of the order of the size of the surface roughness, solid-solid contact may occur through the lubrication layer by means of surface roughness elements. Barnocky \& Davis (1989) extended the analysis of Davis et al. (1986) to include the variation of the density and viscosity with pressure. The increase in density and viscosity with pressure results in solidification of the fluid in the contact area, significantly affecting the deformation of an elastic particle and enhancing the rebound. Barnocky $\&$ Davis (1989) proposed a piezoviscous lengthscale $x_{3}=\left(\hat{\eta} \mu_{0} v_{0} R\right)^{1 / 2}$, where $\hat{\eta}$ is a pressure-viscosity coefficient (Chu \& Cameron 1962) and $\mu_{0}$ is the viscosity at the reference pressure. For gap separations smaller than $x_{3}$, piezoviscous effects cannot be neglected.

Several experimental studies have analysed the rebound of a particle colliding with a wall overlaid with a viscous fluid (Barnocky \& Davis 1988; Lundberg \& 
Shen 1992; Davis, Rager \& Good 2002) or with a fully immersed wall (McLaughlin 1968; Gondret et al. 1999; Joseph et al. 2001; Gondret, Lance \& Petit 2002). In all cases, the results show a minimum particle Stokes number $S t_{c}$ below which no rebound occurs. A comparison between particle image velocimetry measurements and lattice-Boltzmann simulations was made by ten Cate et al. (2002). Their experiments were all carried out at $S t<S t_{c}$ and consequently no rebound was observed. In a related study by Zhang et al. (1999), spheres were dropped onto a stationary sphere in a liquid such that both collinear and oblique collisions could be achieved. Their measurements were favourably contrasted with lattice-Boltzmann simulations and with a mechanistic model that describes the collision process.

With the exception of the study by Zhang et al. (1999), all of the above-mentioned experimental results were obtained with normal or near-normal collisions. A recent study by Kantak \& Davis (2004) addresses oblique collisions with a wall overlaid with a viscous fluid. Their results show the tangential component of the motion to be essentially unchanged by the collision and the normal component of the motion to be a function of a modified Stokes number based on the normal impact velocity. Sundin \& Åhrström (1999) and Workel et al. (2001, 2003) developed ball impact apparatuses for measuring the friction coefficients of lubricated surfaces subject to transient EHD loads at very high pressures. The results from both these research groups are in agreement. Åhrström, Penchinat \& Norrby (2003) improved the method used by Sundin \& Åhrström (1999) to provide a qualitative explanation of the link between inserted energy and the thermal properties of a lubricant.

The present oblique-collision study is an extension of the normal-collision work of Joseph et al. (2001). High-speed video images of immersed oblique collisions are used to characterize the normal restitution and frictional losses of rough and smooth particles impacting a smooth wall. An explanation of the observed behaviour based on the results presented by Joseph et al. (2001) and on tribology is presented.

\section{Oblique impact of spheres}

The oblique impact of an elastic sphere and a flat surface can be analysed based on Hertzian contact theory (Timoshenko \& Goodier 1970). According to Hertzian theory, the maximum radius of contact $a$ of a sphere normally impacting an elastic half-space at a velocity $V$ is given by

$$
a=\left(\frac{3 R W}{4 E^{*}}\right)^{1 / 3},
$$

where $W$ is the equivalent load due to the impact and is obtained from

$$
W=\frac{4}{3} R^{2} E^{*}\left(\frac{5 \pi}{4 E^{*}} \rho_{p} V^{2}\right)^{3 / 5} .
$$

An oblique collision, such as that shown in figure 1, can be considered as a superposition of the normal and tangential components of relative motion, with the interaction in the normal direction described by Hertzian theory. Two distinct interaction mechanisms are recognizable in the tangential direction: sliding and rolling. In sliding interactions, the relative velocity of the contacting surfaces is nonzero. In rolling interactions, the surfaces at the point of contact do not move with respect to each other even if the relative velocity of the centres of mass of the contacting objects is non-zero. 


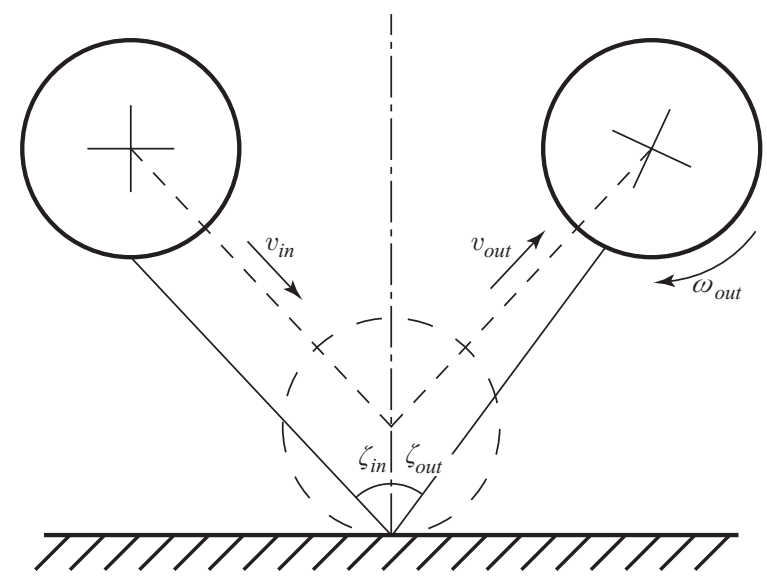

FIGURE 1. Schematic representation of the oblique collision of a sphere with a flat surface. The sphere approaches the wall with no spin. The solid and dashed lines indicate the trajectories of the contact point and mass centre, respectively.

Assume momentarily that tangential interaction between the sphere and the surface is not significantly affected by the elastic deformation of the surfaces in the normal direction. Further assume that the sphere begins to slide in a direction parallel to the wall. If the contact surfaces are not ideally smooth, the sphere experiences an opposing force as a consequence of friction. For a large enough frictional force, the relative velocity of the contacting surfaces drops to zero while the velocity of the centre of mass of the sphere is non-zero. From that instant until the end of the collision, the sphere rolls on the flat surface.

In a physical system, the elastic deformation of the contacting surfaces cannot be neglected. Mindlin (1949) studied the case where two homogeneous isotropic elastic bodies are pressed together with a constant force $W$ and then subjected to a tangential force $F$. If $F>\mu_{f} W$, where $\mu_{f}$ is a constant coefficient of friction, gross sliding occurs throughout the contact area, whereas, for lower values of $F$, Mindlin concluded that a circular inner region of the contact area remains stuck while a surrounding annulus slides or micro-slips. It should be pointed out that the contact region is not necessarily circular. Cattaneo (1938) derived the equations corresponding to elliptic contact surfaces. These equations are analogous to the equations for circular contacts and, in general, the circular contact approximation is of satisfactory accuracy.

This approach has the problem of assuming a constant load $W$ throughout. As colliding particles come into contact, deform, and eventually rebound, the load conditions are not that simple. This limitation was addressed by Mindlin \& Deresiewicz (1953), who proposed a method for solving cases where the normal and tangential loads vary. The approach described by Mindlin \& Deresiewicz is applicable to a wide variety of collisions. Their result is valid for the case where the spheres are first compressed normally and then sheared. Walton (1978) studied the case where the two motions occur simultaneously. Under those conditions, Walton concluded that if slip occurs, then it will be in the form of sliding, i.e. slip over the whole of the contact area. Elata (1996) warned against misusing the force-displacement relations obtained by Walton. These relations are path-dependent, and are derived assuming a fixed ratio between the normal and tangential displacements of contact. When used to describe certain cyclic contact displacements, Walton's relations predict a net 
energy generation without any work being done, which violates the second law of thermodynamics.

Since the loading history depends on both the compliance of the contact and the motion of the particles, the problem is well suited to a numerical solution that steps forward in time. Maw, Barber \& Fawcett (1976) developed such a method. The contact region is divided by a series of equi-spaced concentric circles which define the limits of a series of tangential traction distributions. A provisional division into slip and stick regions can be assumed. In slip regions, the tangential traction is given by the friction coefficient and the local normal contact pressure distribution. In stick regions, the tangential displacement is prescribed.

In order to achieve more generality, Maw et al. (1976) proposed two nondimensional parameters, applicable to all materials and experimental conditions. The first, $\chi$, is a modified radius of gyration and is defined by

$$
\chi=\frac{(1-v)\left(1+1 / K^{2}\right)}{2-v},
$$

where $v$ is Poisson's ratio and $K$ is a non-dimensional radius of gyration; for a homogeneous sphere, $K^{2}=2 / 5$. The second parameter, $\psi$, is a non-dimensional local angle of contact, and is defined by

$$
\psi\left(v_{T}\right)=\frac{2(1-v) v_{T}}{\mu_{f}(2-v) v_{N, \text { in }}} .
$$

The translational velocity $v$ of the mass centre and the angular velocity $\omega$ about it are shown in figure 1 . The quantity $v_{T}=v+\omega R$ is the instantaneous value of the tangential velocity at the point of contact; $v_{N, i n}$ represents the incident normal velocity of the point of contact. Non-dimensional angles of incidence and reflection, $\psi_{\text {in }}$ and $\psi_{\text {out }}$, can be determined for corresponding impact and rebound tangential velocities of the point of contact, $v_{T, \text { in }}$ and $v_{T, \text { out }}$. A positive angle of reflection is one in which the tangential velocity of the point of contact retains the same sense. The parameter $\psi$ can be interpreted physically with respect to the radius of gyration $\chi$ as follows. For values of $\psi \leqslant 1$, which correspond to small angles of incidence, the normal load is considerably larger than the tangential force of the particle and the surfaces stick during contact, although some micro-slip may occur in the periphery of the contact area where the normal load is markedly lower. For values of $\psi$ between 1 and $(4 \chi-1)$, corresponding to intermediate ranges of the incidence angle, the collision starts in gross slip. In this range, the traction produced by friction is large enough for the sliding velocity to drop to zero before the end of the collision, and the process transitions instantaneously from gross slip to full stick. Collisions at larger angles, where $\psi \geqslant(4 \chi-1)$, occur entirely in gross slip.

A drawback of this otherwise general approach is that, in order to evaluate $\psi$ from experimental measurements, a prior quantitative evaluation of the coefficient of sliding friction is required. At the beginning of a collision, the only independent input variable for a given system is the effective angle of incidence, with the material properties captured by $\chi$ and $\mu_{f}$. The local angles of incidence, $\zeta_{\text {in }}$, and rebound, $\zeta_{\text {out }}$, at the contact area, shown in figure 1 , are given by

$$
\begin{gathered}
\tan \zeta_{\text {in }}=\frac{v_{T, \text { in }}}{v_{N, \text { in }}}=\frac{\mu_{f}(2-v)}{2(1-v)} \psi_{\text {in }} \equiv \Psi_{\text {in }}, \\
\tan \zeta_{\text {out }}=\frac{1}{e} \frac{v_{T, \text { out }}}{v_{N, \text { in }}}=\frac{1}{e} \frac{\mu_{f}(2-v)}{2(1-v)} \psi_{\text {out }} \equiv \frac{1}{e} \Psi_{\text {out }},
\end{gathered}
$$


and are used to define effective angles of incidence and rebound, $\Psi_{\text {in }}$ and $\Psi_{\text {out }}$. The normal coefficient of restitution, $e$, is defined by the ratio of the rebound to impact normal velocities of the point of contact, $e=-v_{N, \text { out }} / v_{N, i n}$.

Instead of evaluating a continuously varying force-displacement relationship for the collision of elastic particles, it is often preferable to specify an instantaneous collision operator for the equivalent system of rigid particles. Walton (1993) presents a simplified description of collisions between macroscopic spheres by introducing a three-parameter model:

(a) a normal coefficient of restitution, $e$;

(b) a rotational coefficient of restitution, $\beta$, for contacts that are not continuously sliding during the entire collision; and

(c) a coefficient of sliding friction, $\mu_{f}$, for sliding or grazing collisions.

The rotational coefficient of restitution, defined by the ratio of the rebound to impact tangential velocities of the point of contact, $\beta=-v_{T, o u t} / v_{T, i n}$, is the same as the roughness coefficient $\beta$ introduced by Lun \& Savage (1987). Unlike the description by Maw et al. (1976), Walton's model implies that slipping and sticking regions do not coexist in a given impact.

Application of this method leads to a sliding solution of the form

$$
\Psi_{\text {out }}=\Psi_{\text {in }}-\mu_{f}(1+e)\left(1+1 / K^{2}\right) .
$$

For non-sliding collisions, the obtained solution is

$$
\Psi_{\text {out }}=-\beta \Psi_{\text {in }} .
$$

Since, in Walton's model, $\mu_{f}$ and $\beta$ are mutually exclusive properties of the contact point, it is not possible to extract either of their values from an individual collision without knowing beforehand whether or not the collision involves gross sliding. This limitation can be circumvented by plotting $\Psi_{\text {out }}$ as a function of $\Psi_{\text {in }}$. From such a plot, the slope of (2.7) and the intercept of (2.6) can be used to determine $\beta$ and $\mu_{f}$, respectively (Maw, Barber \& Fawcett 1981; Foerster et al. 1994),

$$
\begin{gathered}
\beta=-\frac{\Psi_{\text {out }}}{\Psi_{\text {in }}}, \\
\mu_{f}=\frac{\Psi_{\text {in }}-\Psi_{\text {out }}}{(1+e)\left(1+1 / K^{2}\right)} .
\end{gathered}
$$

Equation (2.8) can be considered valid for the entire range of collisions, from sliding to sticking (or rolling). In that sense, a value of $\beta=-1$ indicates full nondissipative sliding, since it results from identical $v_{T, \text { in }}$ and $v_{T, \text { out }}$. The case where $\beta=0$ corresponds to a rolling contact where $v_{T, \text { out }}=0$. A value of $\beta=1$ suggests a collision with full recoil, where $v_{T, \text { out }}=-v_{T, i n}$. Non-zero values between these extrema indicate dissipation during the collision, either frictional losses for sliding collisions or inelasticity in the contact for collisions with recoil.

A plot of (2.9) for the entire range of collisions has two distinct regions. The first region, where the calculated $\mu_{f}$ steadily grows towards a plateau, corresponds to non-sliding collisions. The second region is the plateau itself, where a fairly constant value of $\mu_{f}$ indicates sliding collisions. By considering $\beta$ as a variable parameter valid for the entire range of collision angles $\zeta$, equations (2.8) and (2.9) can be rewritten as

$$
\mu_{f}=\frac{2(1+\beta)}{7(1+e)} \Psi_{i n},
$$




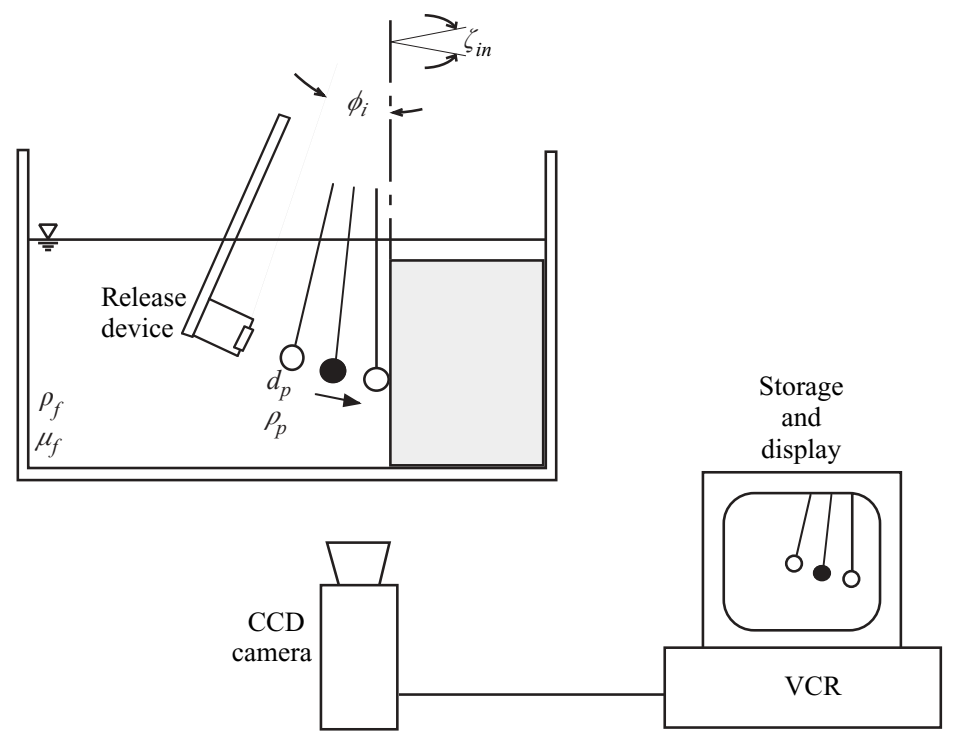

FIGURE 2. The experimental set-up.

$\begin{array}{lccccc}\text { Material } & \begin{array}{c}\rho \\ \left(\mathrm{kg} \mathrm{m}^{-3}\right)\end{array} & \begin{array}{c}E \\ (\mathrm{GPa})\end{array} & v & \begin{array}{c}\sigma_{s} \\ (\mu \mathrm{m})\end{array} & \begin{array}{c}\lambda_{p} \\ (\mu \mathrm{m})\end{array} \\ \text { Glass } & 2540 & 60 & 0.23 & 0.1305 & 22.59 \\ \text { Steel } & 7780 & 190 & 0.27 & 0.0236 & 48.04 \\ \text { Zerodur } & 2540 & 91 & 0.24 & 0.0155 & 1075\end{array}$

TABle 1. Properties of materials used in collision experiments.

where $K^{2}=2 / 5$ has been used. The coefficients of restitution $e$ and $\beta$ and the incidence angle $\Psi_{i n}$, from which $\mu_{f}$ can be calculated, are all measurable quantities based on the tangential and normal velocities of the point of contact.

\section{Dry collisions}

To validate the applicability of the pendulum set-up of Joseph et al. (2001) for the measurement of oblique collisions, experiments were performed with a $12.7 \mathrm{~mm}$ steel ball-bearing and a $12.7 \mathrm{~mm}$ glass sphere impacting a Zerodur block in air. The experimental set-up is shown in figure 2. The densities, Young's moduli, and Poisson's ratios of these materials are presented in table 1 . The table also includes the root-mean-square surface roughness $\sigma_{s}$ and correlation length $\lambda_{p}$ of the particles, as obtained from scanning electron microscopy images by Joseph et al. (2001), and of the Zerodur block, measured using a stylus profilometer.

The azimuthal orientation of the wall was adjusted such that its normal formed an angle $\zeta_{\text {in }}$ with respect to the plane of the pendulum. The motion of the spheres was filmed using a high-speed digital camera with a framing rate of 1000 frames per second. The resulting digital movie was processed to determine the position of the centroid of the particle in each frame, as described by Joseph et al. (2001). To determine the angular velocity of the particle, two dots were painted at diametrically opposing positions, such that the high-speed camera could see both dots. The positions 


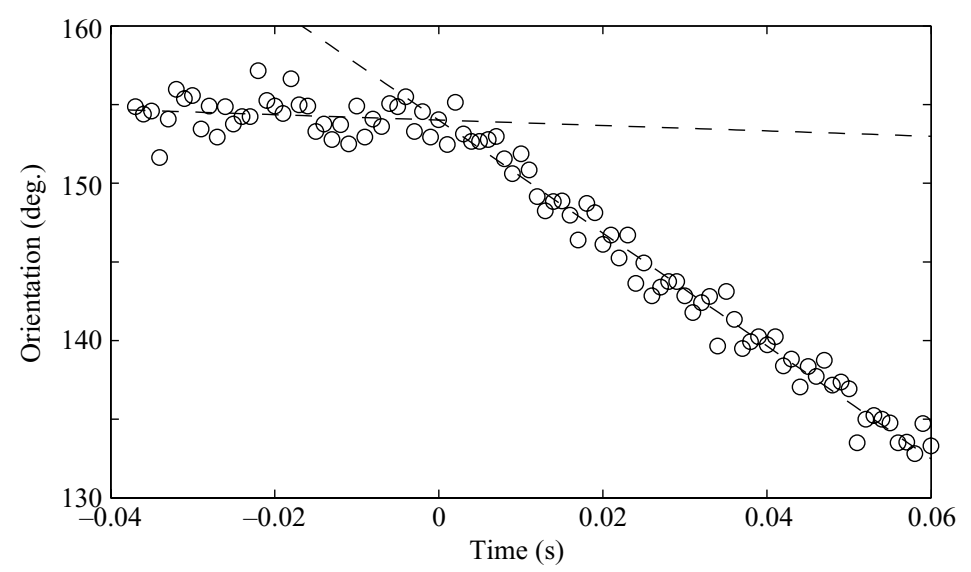

FIGURE 3. Particle position and velocity for a $12.7 \mathrm{~mm}$ glass particle in an aqueous solution of glycerol $(45 \% \mathrm{wt})$ impacting the Zerodur wall at an angle $\zeta_{\text {in }}=20^{\circ}$. The angular velocities before and after impact are $0.3 \mathrm{rad} \mathrm{s}^{-1}$ and $6.26 \mathrm{rad} \mathrm{s}^{-1}$, respectively. For this collision, the normal coefficient of restitution is $0.708(R e=374, S t=95)$.

of these dots were tracked when processing the movie. The angular orientation of the particle was then determined from the slope of a line joining the tracked dots. By construction, all rotation of the spheres occurs around the axis of the pendulum string. For the present experiments, the torsional stiffness of the string is negligible, as shown in the Appendix.

Figure 3 is an example of angular orientation versus time obtained from the rotation tracking. The data correspond to a $12.7 \mathrm{~mm}$ diameter glass sphere released from an angle of $\phi_{i}=18^{\circ}$ supported by a line of length $10 \mathrm{~cm}$. The plane of the pendulum forms an angle $\zeta_{\text {in }}=20^{\circ}$ with the normal of the Zerodur target. The time $t=0$ corresponds to the collision time and is determined from the translational velocity measurements (Joseph et al. 2001). The angular velocities before and after the collision are determined from the slopes of the angular orientation versus time data.

Figure 4 shows the comparison between the collisions of a $12.7 \mathrm{~mm}$ steel sphere and a $12.7 \mathrm{~mm}$ glass sphere impacting a Zerodur wall, corresponding to a range of normal incident velocities of $50-380 \mathrm{~mm} \mathrm{~s}^{-1}$. Velocities in this range are smaller than the velocity corresponding to the fully plastic impact region for which the coefficient of restitution has been shown to decrease with increasing impact velocity (Goldsmith 1960; Johnson 1985). The mean normal coefficients of restitution of these collisions are $\bar{e}_{d r y}=0.97 \pm 0.02$ for both cases, in agreement with the values measured by Joseph et al. (2001). The measurements made using the pendulum set-up in air compare favourably with the experiments of Maw et al. (1981), where pucks on an air table were made to collide with a polished steel block of matching specification. The pucks used by Maw et al. were sliced by spark erosion from commercial steel ball-bearings $101.6 \mathrm{~mm}$ in diameter.

Linear fits to the $\Psi_{\text {out }}$ versus $\Psi_{\text {in }}$ data, based on (2.8) and (2.10), are also shown in figure 4. From these fits, the values of the rotational coefficient of restitution ( $\beta=0.34 \pm 0.07$ for steel, $\beta=0.39 \pm 0.06$ for glass) and the coefficient of sliding friction $\left(\mu_{f}=0.11 \pm 0.003\right.$ for steel, $\mu_{f}=0.106 \pm 0.008$ for glass $)$ were calculated. The reported uncertainties correspond to the correlation values of the line fits. These 


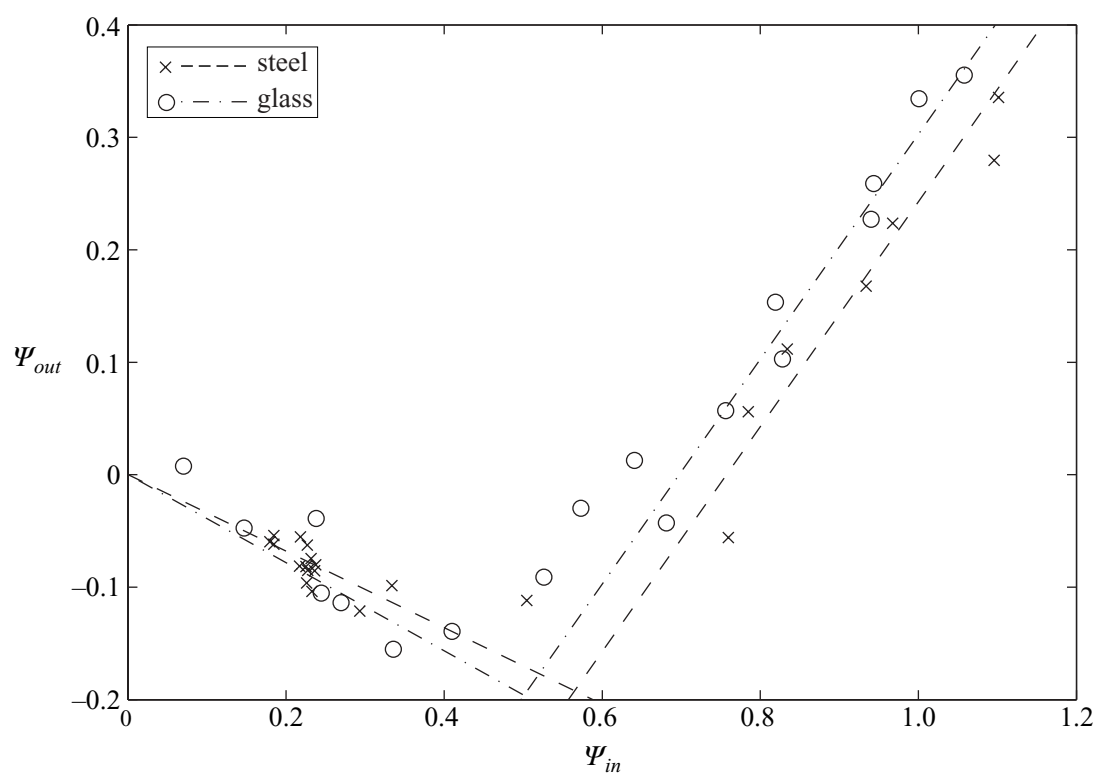

Figure 4. Comparison between the oblique collision of a $12.7 \mathrm{~mm}$ steel sphere and a $12.7 \mathrm{~mm}$ glass sphere with a Zerodur wall in air. The lines indicate linear fits corresponding to equations (2.8) and (2.10).

values of $\beta$ and $\mu_{f}$ are consistent with those measured by Maw et al. (1981) and Foerster et al. (1994).

\section{Collisions in a liquid}

Measurements were obtained for collisions of glass and steel spheres on the Zerodur wall in glycerol-water mixtures at temperatures of approximately $20^{\circ} \mathrm{C}$. Figure 5 shows the normal coefficient of restitution $e$ obtained from these measurements. The shaded band corresponds to the measurements of normal collisions by Joseph et al. (2001). The data are plotted as a function of a modified Stokes number, St $\cos \zeta_{\text {in }}=2 \rho_{p} R v_{N, \text { in }} / 9 \mu$, based on the normal component of the impact velocity. The incident angle $\zeta_{\text {in }}$ is the angle formed by the trajectory of the contact point and the wall's normal. The trend is the same for all cases, regardless of the incident angle. Within experimental uncertainty, the data show the normal coefficient of restitution in oblique collisions to be independent of the tangential component of velocity. The results from purely normal collisions, like those presented by Joseph et al. (2001), may be used directly in (2.10) when characterizing oblique immersed collisions.

Figure 6 shows three sets of experimental data for collisions of a $12.7 \mathrm{~mm}$ glass sphere with a Zerodur wall. The corresponding surrounding fluids are water $(1 \mathrm{cP})$ and two aqueous solutions of glycerol (33\% wt, $2.5 \mathrm{cP} ; 45 \% \mathrm{wt}, 4.5 \mathrm{cP})$. Each data point indicates the average value of five distinct experimental runs performed for nominal angles $\zeta_{i n}$, and the error bars represent the standard deviation of the five averaged measurements. The rich behaviour of these collisions is qualitatively and quantitatively similar to that observed in the experiments of Maw et al. (1981) and Foerster et al. (1994), and also to the dry collision data shown in figure 4. The dotted line indicates the locus of a frictional coefficient $\mu_{f}=0.11$, corresponding to the value obtained in $\S 3$. For small values of $\Psi_{i n}$, a positive value of the non-dimensional 


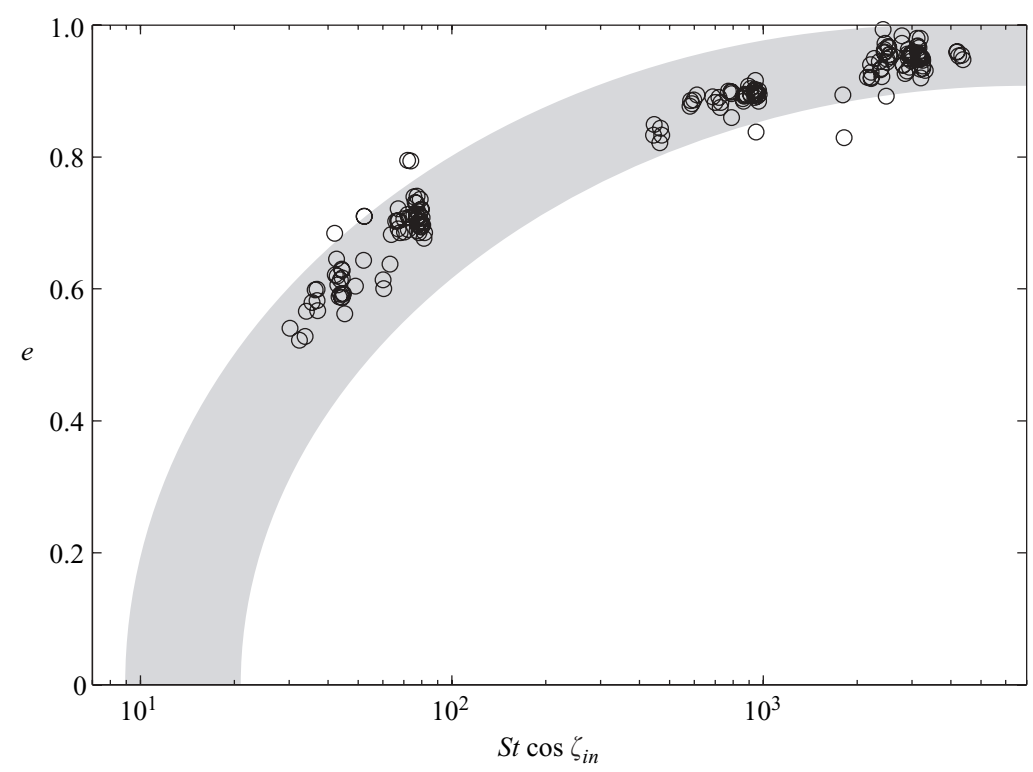

FIGURE 5. Effective normal coefficient of restitution, $e$, as a function of a modified Stokes number based on the normal velocity of impact, St $\cos \zeta_{\text {in }}$, for immersed oblique collisions in water and glycerol-water mixtures. The shaded band shows the region occupied by the experimental results from normal collisions reported by Joseph et al. (2001).

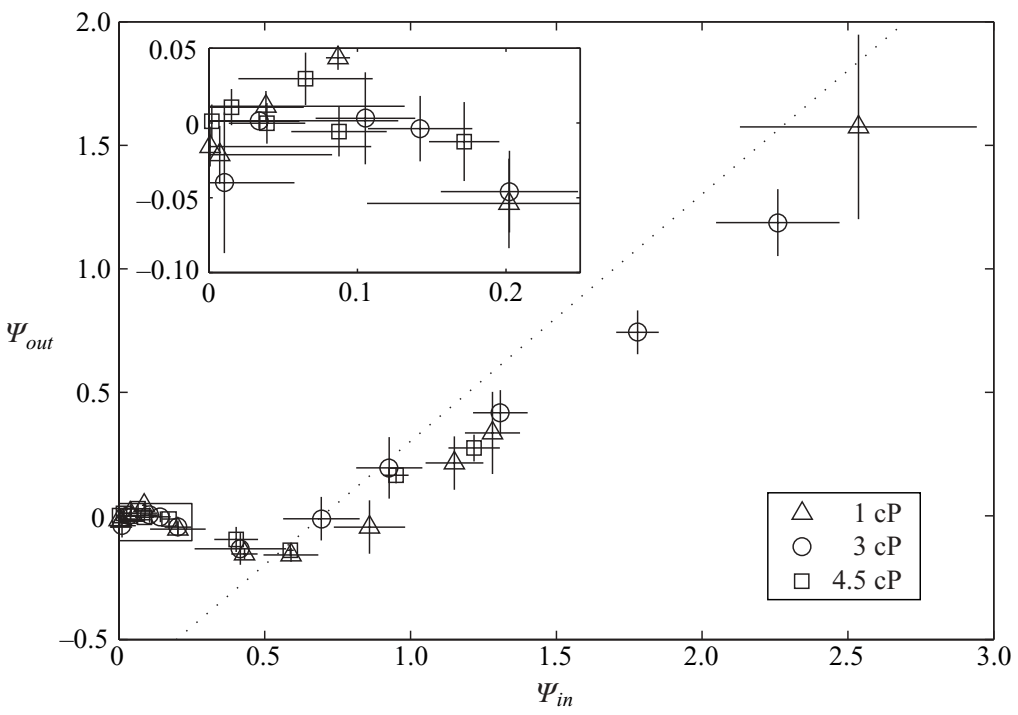

FIGURE 6. Non-dimensional rebound angle as a function of the non-dimensional incidence angle for a $12.7 \mathrm{~mm}$ glass sphere impacting obliquely without spin on a Zerodur wall. The surrounding fluids are $\triangle, 1 \mathrm{cP}, \bigcirc, 2.5 \mathrm{cP}$ and $\square, 4.5 \mathrm{cP}$ aqueous solutions of glycerol. Each point represents the average value of five distinct experimental runs. The error bars on each point indicate the standard deviation of the five measurements. The dotted line indicates the locus of a frictional coefficient $\mu_{f}=0.11$. 


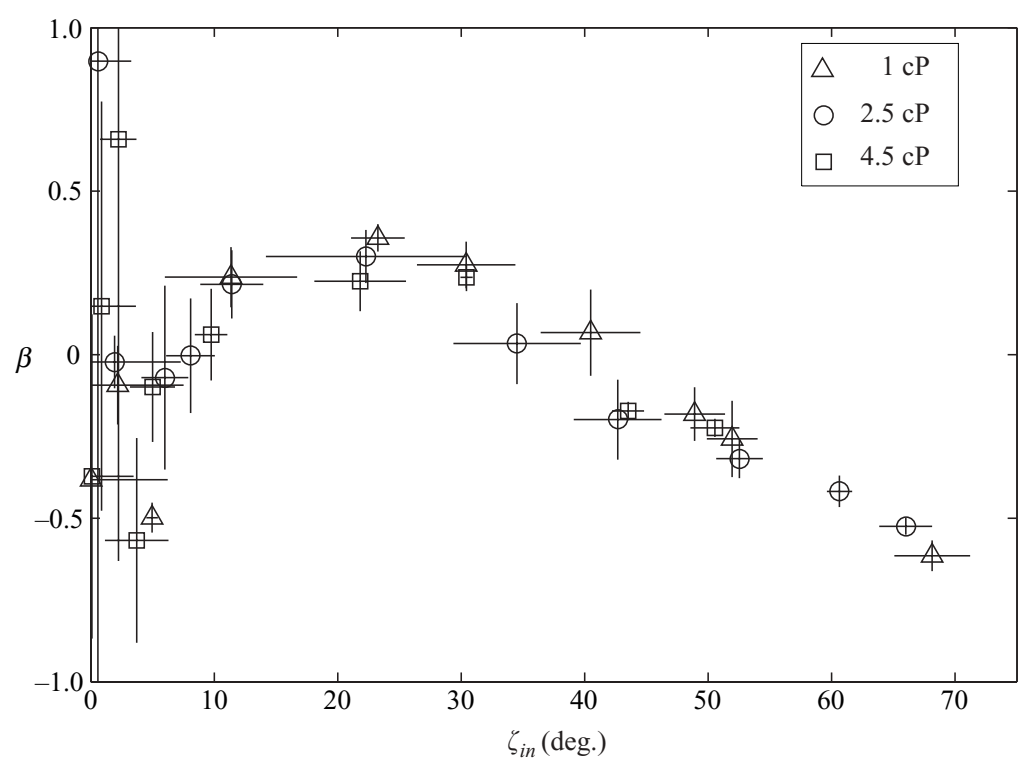

FIGURE 7. Rotational coefficient of restitution corresponding to the data from figure 6.

rebound angle is observed (see inset of figure 6), a consequence of micro-slip at the beginning of the impact, as identified by Maw et al. (1976). The collisions with $\Psi_{\text {in }}$ from 0.1 to 0.6 , corresponding to impact angles from $5^{\circ}$ to $30^{\circ}$, exhibit negative values of $\Psi_{\text {out }}$, indicative of recoil in the collision. Such recoil is presumably a consequence of solid-solid contact. For glass spheres, the mean surface roughness is larger than the EHD lubrication minimum distance of approach $h_{m}$ (Joseph et al. 2001). Therefore, interaction between the asperities is to be expected. For $\Psi_{\text {in }} \approx 0.6\left(\zeta_{\text {in }}=30^{\circ}\right)$ and higher, gross sliding occurs throughout the collision. A relatively large scatter is exhibited by the data beyond $\Psi_{i n} \approx 1$. For these collisions, any slight variation in the incidence angle $\zeta_{\text {in }}$ has a large effect on the values of $\Psi_{\text {in }}$ and $\Psi_{\text {out }}$, since these parameters are functions of $\tan \zeta_{\text {in }}$ (see (2.5)).

A plot of the derived rotational coefficient of restitution, based on (2.8), is presented in figure 7. The rotational coefficients of restitution for near-normal collisions correspond to a large error band because the rotation is small. Two regions can be identified on the plot of $\beta$. The middle data points, where $\beta>0\left(\zeta_{\text {in }}\right.$ between $5^{\circ}$ and $30^{\circ}$ ), correspond to collisions where recoil is observed. For these points, the computed coefficient of friction is steadily rising and it can be interpreted as being proportional to the tangential force necessary to balance the tangential elastic compliance of the colliding objects. The rightmost points, with $\beta<0\left(\zeta_{\text {in }}>30^{\circ}\right)$, correspond to sliding cases where the frictional force between the surfaces is no longer enough to balance the tangential motion of the sphere. Shown in figure 8 is the coefficient of sliding friction, calculated from (2.9). Experimentally determined values of the coefficient of restitution $e$, calculated for each data point from the corresponding normal components of velocity, were used when computing $\mu_{f}$. The plot shows that for near-normal collisions there is no sliding, as anticipated from figure 6. For higher incidence angles, the computed coefficient of friction is $\mu_{f}=0.15 \pm 0.03$, somewhat higher than that found for the dry collisions, but quantitatively consistent with values reported in the literature (cf. Avallone \& Baumeister 1987). 


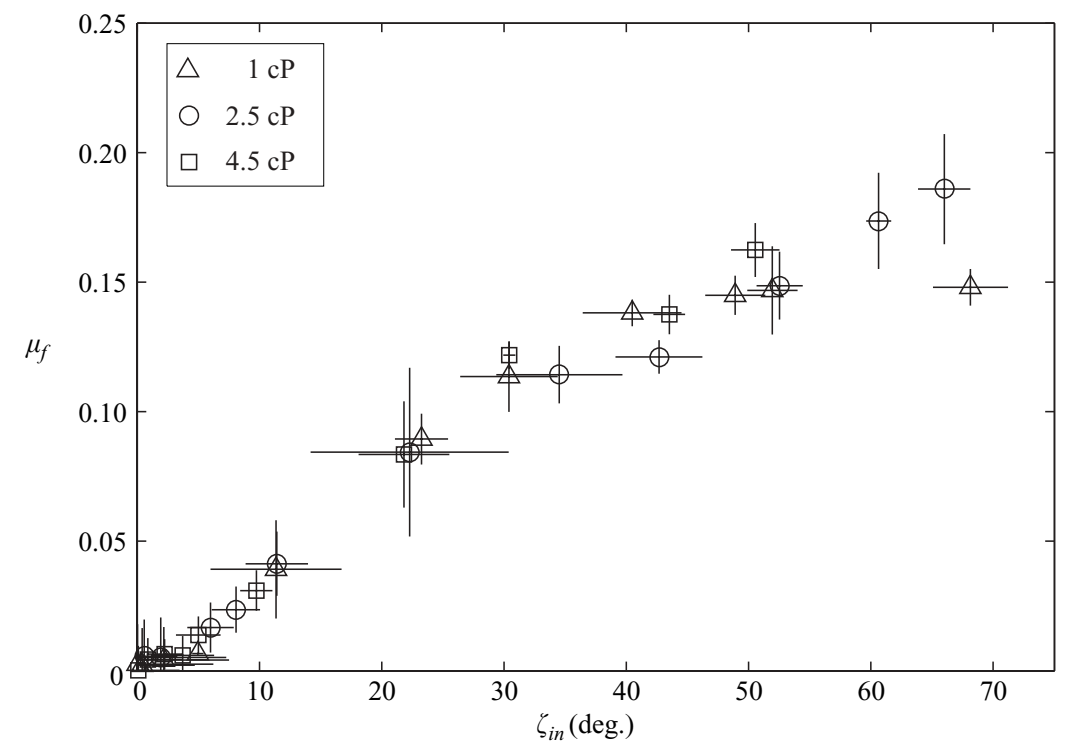

FIGURE 8. Computed effective coefficient of friction corresponding to the data from figure 6 .

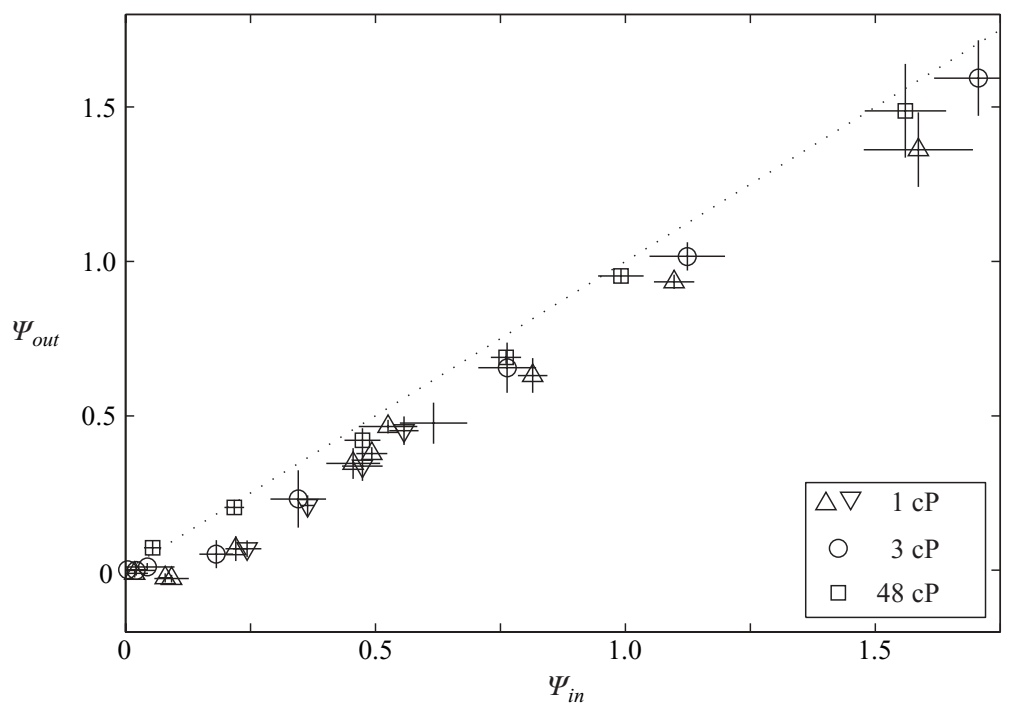

FIGURE 9. Non-dimensional rebound angle as a function of the non-dimensional incidence angle for a $12.7 \mathrm{~mm}$ steel sphere impacting obliquely without spin on a Zerodur wall. The surrounding fluids are $1 \mathrm{cP}, 3 \mathrm{cP}$ and $48 \mathrm{cP}$ aqueous solutions of glycerol. Each point represents the average value of five distinct experimental runs. The error bars on each point indicate the standard deviation of the five measurements. The dotted line indicates the locus of a specular rebound with friction coefficient $\mu_{f}=0$. The different triangles correspond to pendulum string diameters of $127 \mu \mathrm{m}(\triangle)$ and $51 \mu \mathrm{m}(\nabla)$.

Experiments were also performed with steel particles in water $(1 \mathrm{cP})$ and in glycerolwater solutions with concentrations of $37 \%$ and $78 \%$ by weight. Those concentrations correspond to viscosities of $3 \mathrm{cP}$ and $48 \mathrm{cP}$, respectively. The results of those collisions are shown in figure 9. Compared with the results for dry collisions or for collisions 


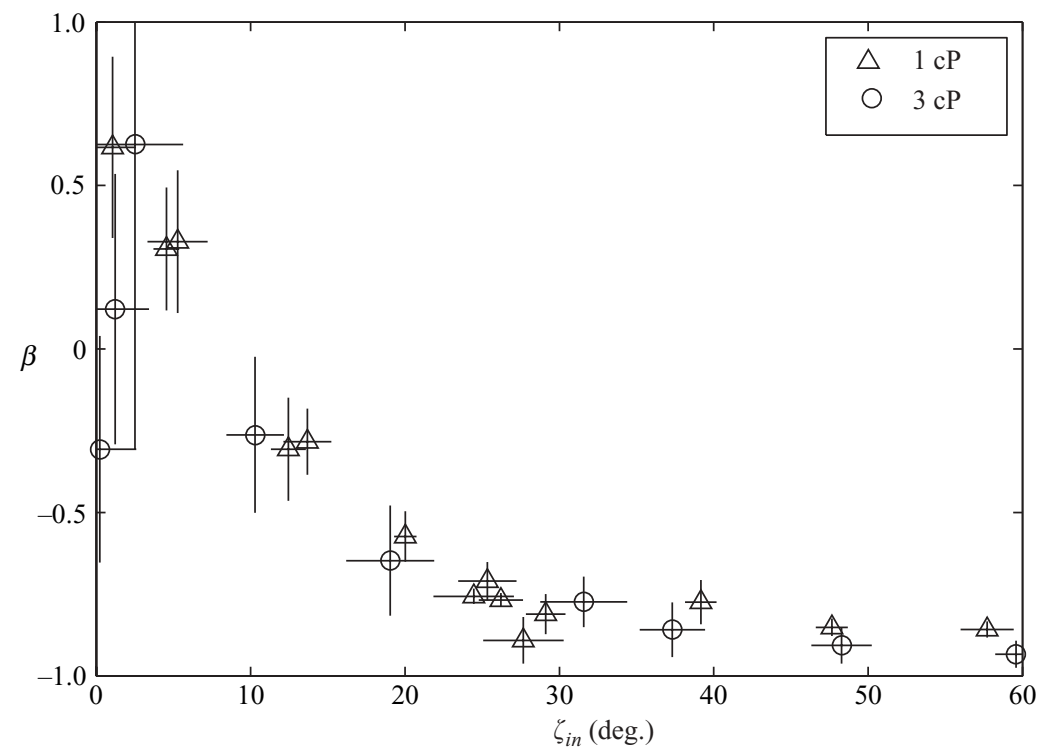

FIGURE 10. Rotational coefficient of restitution corresponding to the data from figure 9.

with glass particles, there is little incursion of the data into negative values of $\Psi_{\text {out }}$, which indicates an absence of recoil in the collisions. The dotted line, of slope one, is the theoretical trajectory of a specular rebound with no rotation, and is provided for comparison purposes. The upright triangles $(\triangle)$ correspond to measurements obtained with a pendulum made using a $\varnothing 127 \mu \mathrm{m}$ string. To ascertain that the torsional stiffness of the pendulum string is negligible, a set of measurements was carried out using a $\varnothing 51 \mu \mathrm{m}$ string. The results obtained from those measurements, shown in figure 9 with upside-down triangles $(\nabla)$, are indistinguishable from results obtained with the $\varnothing 127 \mu \mathrm{m}$ string. Therefore, only upright triangles will be used in subsequent figures, regardless of string diameter.

The experimental data for water clearly follow a slope-one line for $\Psi_{\text {in }}>0.2$ with a negative intercept on the $\Psi_{\text {out }}$ axis. The data for the $3 \mathrm{cP}$ ambient fluid illustrate the same qualitative behaviour as the data for water. Note that the experimental points in this case are closer than those from the collisions in water to the line indicating specular reflection. The slope of the data has a consistent value of one for collisions beyond $\Psi_{i n}=0.15$. The data for the $48 \mathrm{cP}$ surrounding liquid lie close enough to the line of specular reflection to be practically indistinguishable from a purely sliding collision. For that reason, the data for $48 \mathrm{cP}$ are not included in the following two plots of $\beta$ and $\mu_{f}$.

A plot of the derived rotational coefficient of restitution, based on (2.8), is presented in figure 10. The rotational coefficient of restitution for near-normal collisions is $\beta \approx 0.6$, larger than what would be expected from the measurements by Foerster et al. (1994). However, the error associated with the individual measurements grows considerably as the impact angle decreases. The values quickly drop to a value of $\beta \approx-1$, indicative of sliding collisions. Figure 11 shows the coefficient of sliding friction based on (2.9). For each data point, the value of the coefficient of restitution $e$ was experimentally determined. The computed coefficient of friction for the sliding collisions, computed from (2.10), is approximately 0.02 for all incidence angles greater than $10^{\circ}$. This value is approximately one order of magnitude smaller than the 


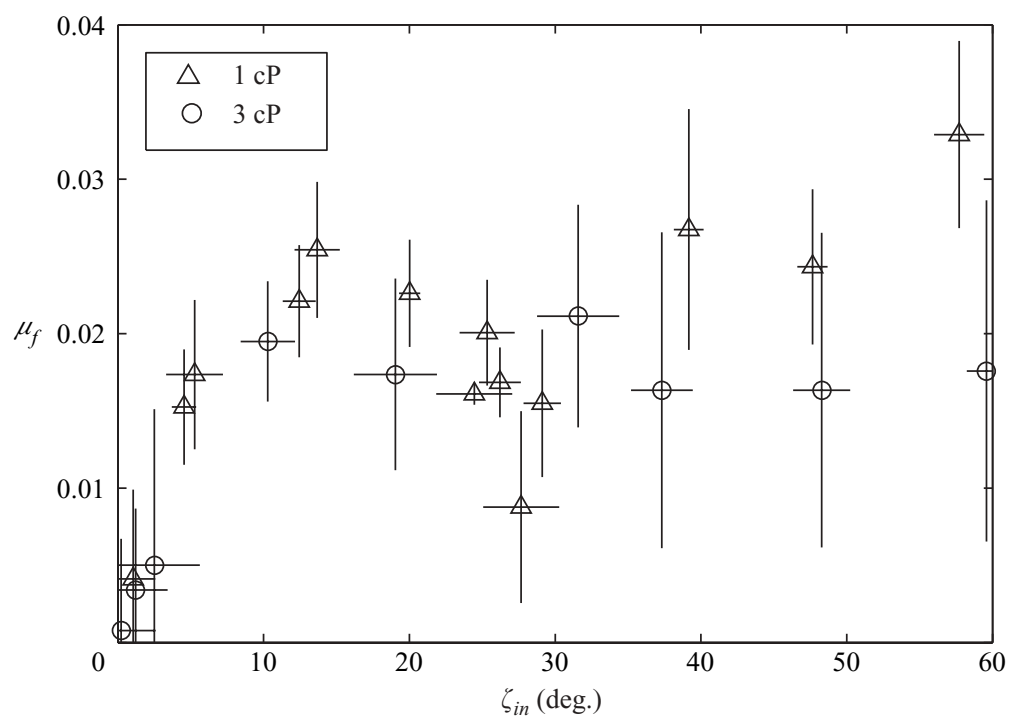

FIGURE 11. Computed effective coefficient of friction corresponding to the data from figure 9.

coefficient of friction for a lubricated contact between steel and glass (Bowden \& Tabor 1950) and is also smaller than the frictional value observed for immersed collisions of glass spheres shown in figure 8.

The different frictional behaviours observed in the glass and steel experiments can be explained based on the surface roughness of those materials. The mean roughness of the glass particles is approximately 2.5 times larger than a minimum gap separation $h_{m}=x_{1} / 3$ (Davis et al. 1986) and about 4 times larger than the piezoviscous lengthscale $x_{3}$ (Barnocky \& Davis 1989). The roughness elements may trap some of the fluid in the lubrication layer and this fluid, owing to piezoviscous effects, may contribute to the higher observed value of the friction coefficient relative to that of dry systems. In the case of the steel spheres, the roughness elements are smaller than the fluid layer; hence, all tangential force experienced by the particle should come from shear in the lubrication layer as discussed in $\S 5$.

Figure 12 shows the local angle of rebound, $\psi_{\text {out }}$, as a function of the local angle of incidence, $\psi_{i n}$, for all the immersed oblique collision experiments. The local angle of contact $\psi$ corresponds to one of the collisional parameters introduced by Maw et al. (1976) and is defined in (2.4). The values shown in figure 12 are a function of $\Psi$ and $\mu_{f}$ and were obtained from (2.5). The individual plots of the effective angles of incidence and rebound shown in figures 6 and 9 collapse onto a single curve in figure 12. The values of the effective angles of incidence and rebound from figure 9 , for collisions with steel in $78 \%$ wt glycerol-water, do not fall on the same curve as the other cases and are not shown here. Note that the experimental error in determining the friction coefficient for this last case is of the order of the friction coefficient itself, making it difficult to distinguish this case from a perfectly sliding collision (for which $\left.\mu_{f}=0\right)$.

\section{Modelling of the fluid effects}

In the preceding section it was shown that, in the presence of solid-solid contact (figure 6, glass spheres), an immersed oblique collision behaves like a dry oblique 


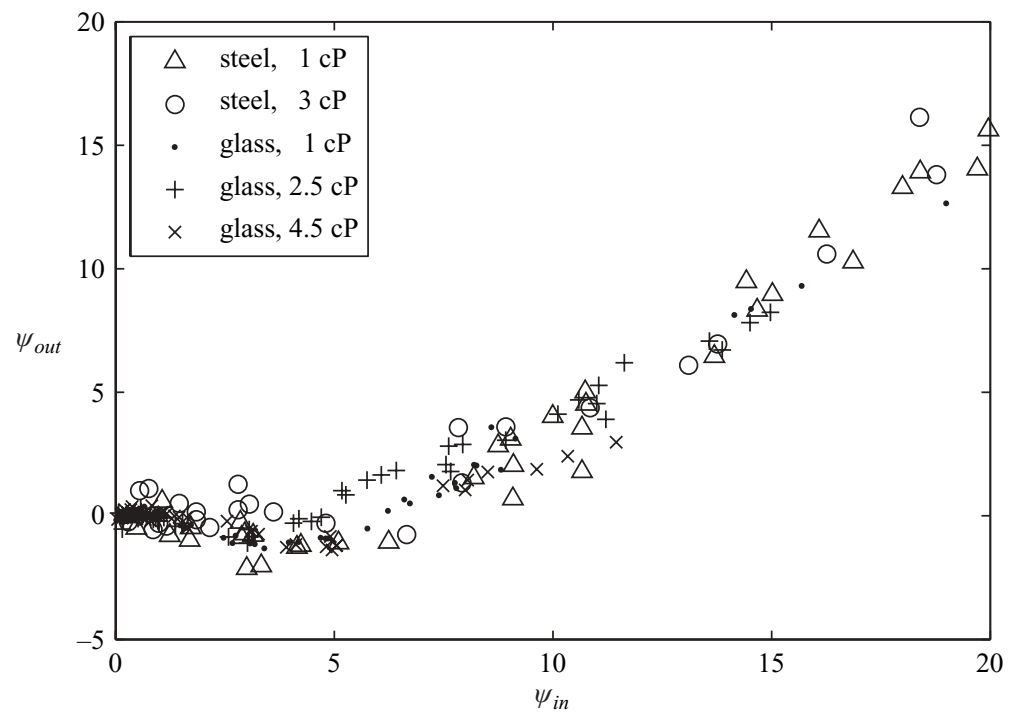

FIGURE 12. Local rebound angle, $\psi_{\text {out }}$, as a function of the local incidence angle, $\psi_{\text {in }}$, for immersed oblique collisions in water and glycerol-water mixtures.

collision. For cases where, owing to EHD lubrication, no solid-solid contact is expected (figure 9, steel spheres), the collisions are qualitatively similar to dry collisions with a relatively small friction coefficient. The friction coefficient, computed from (2.10), falls for most cases within a range of $\mu_{f}$ from approximately 0.001 to 0.1 . In the study of bearings and bushings, this range of frictional force is associated with systems that operate in an EHD lubrication regime (see Cameron 1981). A possible explanation of the observed values of $\mu_{f}$ for the case of immersed collisions follows.

To determine the change in angular velocity of a sphere in an oblique collision, the viscous shear in the gap between the colliding objects must be considered. Christensen (1970) and Barnocky \& Davis (1989) showed that the hydrodynamic pressure that develops during a collision becomes large enough to cause the viscosity of the fluid to increase by several orders of magnitude. In particular, the pressure buildup during the collision process becomes sufficiently large that the corresponding viscosity increase causes the fluid in the gap between two colliding spheres to behave nearly as a solid. Barnocky \& Davis concluded that the effect of the increase in viscosity on the normal component of a collision is minor. In the tangential direction, however, the nearly solidified gap-fluid acts as a point force, imposing a torque on the particle.

A usual way of modelling the pressure dependence on viscosity is to follow Barus's law (Gohar 2001), $\mu=\mu_{0} \exp (C p)$. The pressure-viscosity coefficient $C$ is a constant depending on the fluid, and $\mu_{0}$ is the viscosity at the reference pressure (usually $1 \mathrm{~atm})$. Tabulated values of $C$ are readily available. In some cases, however, Barus's law vastly overpredicts the viscosity. In particular, Gohar (2001) warns against using Barus's law when estimating the friction force of a lubricated contact. A power law pressure-viscosity equation,

$$
\mu=\mu_{0}(1+\hat{\eta} p)^{16},
$$

proposed by Chu \& Cameron (1962), offers a more adequate relation for determining the frictional force. The coefficient $\hat{\eta}$ can be calculated from the pressure coefficient $C$ by matching Barus's law and equation (5.1) at low pressures. 


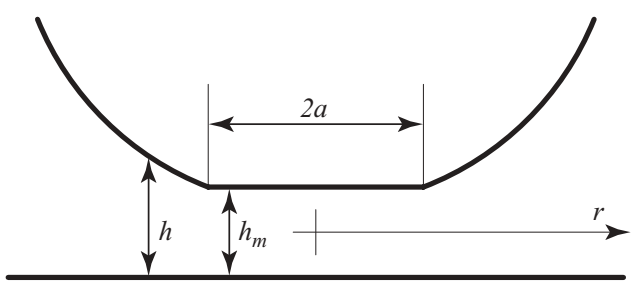

FIGURE 13. Elastohydrodynamic contact of a sphere and a wall. Not to scale.

In order to evaluate the torque imposed on the particle, assume that the deformation of the contact area is Hertzian, as shown in figure 13. The pressure distribution in the gap does not vary throughout the thickness of the fluid film, and is given by Reynolds's equation for pure squeeze motion,

$$
\frac{1}{r} \frac{\partial}{\partial r}\left(r h^{3} \frac{\partial p}{\partial r}\right)=12 \mu \frac{\partial h}{\partial t} .
$$

At this point, it is convenient to define a pressure parameter,

$$
p^{*} \equiv \frac{1-(1+\hat{\eta} p)^{-15}}{15 \hat{\eta}},
$$

based on (5.1). Note that for high pressures $(1+\hat{\eta} p)^{-15} \rightarrow 0$ and $p^{*} \rightarrow 1 / 15 \hat{\eta}$, a constant. Substituting (5.1) into (5.2) and integrating once, the Reynolds equation can be written as

$$
\frac{\partial p^{*}}{\partial r}=\frac{6 \mu_{0} r}{h^{3}} \frac{\partial h}{\partial t}
$$

Within the Hertzian contact region, the pressures are sufficiently high that $p^{*} \rightarrow$ $1 / 15 \hat{\eta}$ and hence $\partial p^{*} / \partial r=0$. From (5.3), it follows that $\partial h / \partial t=0$ and therefore the film thickness is a constant, $h=h_{m}$. Since there is no pressure variation throughout the thickness of the film, the pressure in the contact zone has a Hertzian distribution, $p=p_{\max } \sqrt{1-r^{2} / a^{2}}$. The radial variation of viscosity, from (5.1), is given by

$$
\mu(r)=\mu_{0}\left(1+\hat{\eta} p_{\max } \sqrt{1-r^{2} / a^{2}}\right)^{16} .
$$

In a Hertzian contact, the maximum pressure $p_{\max }$ is given by

$$
p_{\max }=\left(\frac{40}{\pi^{4}} \rho_{p} V^{2} E^{* 4}\right)^{1 / 5} .
$$

Equation (5.3) cannot be used to evaluate the minimum distance of approach $h_{m}$ since one of the initial conditions (the gap separation of incipient deformation, $h=x_{0}$ ) is not known. The prediction of the gap separation presented in $\S 1$ (Davis et al. 1986) ignores the effect of tangential displacement and overestimates the film thickness. A more adequate relation for $h_{m}$ in computing frictional traction (Gohar 2001) is given by the expression

$$
h_{m}=4.3384 R\left(\frac{\mu_{0} U \hat{\eta}}{R}\right)^{5 / 7}\left(\frac{W}{E^{*} R^{2}}\right)^{-1 / 21},
$$

where $U$ is the translational velocity in the direction parallel to the wall and the equivalent load $W$ is given by (2.2). This empirical formula for $h_{m}$, presented by Gohar 


$\begin{array}{cccrc}\begin{array}{c}\text { Glycerol } \\ (\% \text { wt })\end{array} & \begin{array}{c}\theta_{\infty} \\ \left({ }^{\circ} \mathrm{C}\right)\end{array} & \begin{array}{c}\mu_{\infty} \\ (\mathrm{cP})\end{array} & \begin{array}{c}\mu_{0} \\ (\mathrm{cP})\end{array} & \begin{array}{c}\theta_{\text {gap }} \\ \left({ }^{\circ} \mathrm{C}\right)\end{array} \\ 0 & 20.0 & 1 & 0.8 & 30 \\ 37 & 21.5 & 3 & 1.6 & 43 \\ 78 & 20.5 & 48 & 11.5 & 47\end{array}$

TABLE 2. Ambient and corrected gap viscosities and temperatures in EHD contacts.

(2001) in dimensionless form, was obtained from extensive numerical simulations of EHD contacts.

The frictional traction $F$ on the contact area is a consequence of the shear imposed on the gap by the tangential velocity of the sphere, and is given by the expression

$$
F=\frac{2 \pi U}{h_{m}} \int_{0}^{a} \mu(r) r \mathrm{~d} r .
$$

Combining (5.4) and (5.7), the friction coefficient $\mu_{f}=F / W$ takes the form

$$
\mu_{f}=\frac{2 \pi \mu_{0} U}{W h_{m}} \int_{0}^{a}\left(1+\hat{\eta} p_{\max } \sqrt{1-r^{2} / a^{2}}\right)^{16} r \mathrm{~d} r .
$$

Upon computing $\mu_{f}$ from (5.8) for the nominal experimental conditions explored in this study, the calculated values of the friction coefficient are found to be much larger than those determined experimentally from (2.10). This discrepancy occurs because the energy used in overcoming the viscous forces in the liquid is dissipated as heat, and the resulting temperature rise in the film layer significantly reduces the viscosity.

Curves based on (5.8) can be fitted to the measured values for the coefficient of friction by using $\mu_{0}$ as a parameter. Note from (5.6) that $h_{m}$ is a function of $\mu_{0}$. Figure 14 shows the resulting curve fits together with experimental points corresponding to the measurements reported in figure 9. The curves resulting from ignoring any dependence of viscosity on pressure and temperature correspond to extremely low frictional values $\left(\mu_{f} \approx 10^{-4}\right)$ and are not shown. The experimental data for $48 \mathrm{cP}$ ambient liquid, which had been omitted from figure 11 because of their large error band, are shown here for comparison purposes. The corrected viscosity of the fluid $\mu_{0}$, based on these fits, and the corresponding fluid temperature in the gap $\theta_{\text {gap }}$ are reported in table 2 . The ambient temperature $\theta_{\infty}$ and corresponding viscosity $\mu_{\infty}$ for each experimental set are also reported. The gap temperatures determined in this manner range from $30{ }^{\circ} \mathrm{C}$ to $47^{\circ} \mathrm{C}$ and are consistent with those reported by Bowden \& Tabor (1950).

Figures $14(b)$ and $14(c)$ show good agreement between the experimental measurements and the theoretical estimates. For those cases, a large fraction of the surrounding fluid $(37 \%$ and $78 \%$, respectively) is glycerol. The viscosity of glycerol, from the values tabulated in Gohar (2001), is dependent on pressure with a pressure coefficient $C \approx 6 \times 10^{-9} \mathrm{~Pa}^{-1}$. The corresponding coefficient $\hat{\eta}$ in (5.1) is approximately $375 \times 10^{-12} \mathrm{~Pa}^{-1}$. On the other hand, figure $14(a)$ has a region around $\zeta_{\text {in }}=30^{\circ}$ where the experimental values of the friction coefficient are considerably lower than those predicted by (5.8). At pressures up to about $150 \mathrm{MPa}$ and temperatures up to $30{ }^{\circ} \mathrm{C}$, the viscosity of water decreases with pressure (Eisenberg \& Kauzmann 1969). As the pressure increases, water's hydrogen-bonded network of icosahedral water clusters, which is partially responsible for the viscosity of water, becomes deformed and loses strength. This reduction in cohesivity more than compensates for the reduced void 

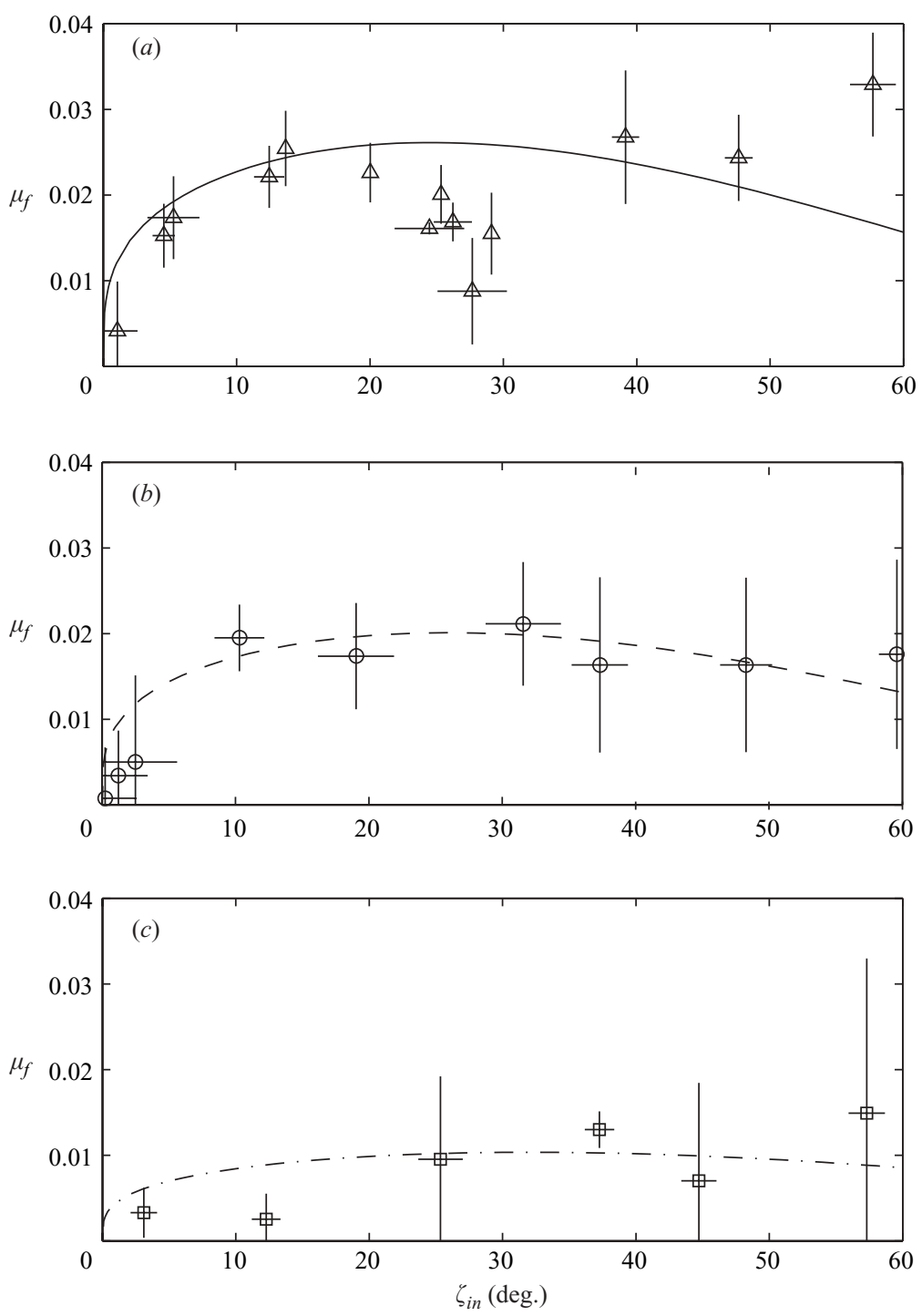

FIGURE 14. Comparison between theoretically calculated and experimentally measured friction coefficient values for collisions of a $12.7 \mathrm{~mm}$ steel ball bearing with the Zerodur wall. The surrounding fluids are $(a)$ water, $(b)$ a $37 \%$ wt aqueous glycerol solution and $(c)$ a $78 \%$ wt aqueous glycerol solution.

volume between the water molecules, hence reducing the viscosity. At higher pressures, water behaves like a normal liquid and its viscosity, a function of the void fraction between the molecules, increases with pressure. With this in mind, the results with water are expected to exhibit a lower value than the prediction from (5.8), which assumes a power-law increase in viscosity with pressure.

The integral in (5.8) can be non-dimensionalized by introducing an integration variable $z=r / a$, such that

$$
\int_{0}^{a}\left(1+\hat{\eta} p_{\max } \sqrt{1-r^{2} / a^{2}}\right)^{16} r \mathrm{~d} r=a^{2} \int_{0}^{1}\left(1+\hat{\eta} p_{\max } \sqrt{1-z^{2}}\right)^{16} z \mathrm{~d} z=a^{2} A
$$




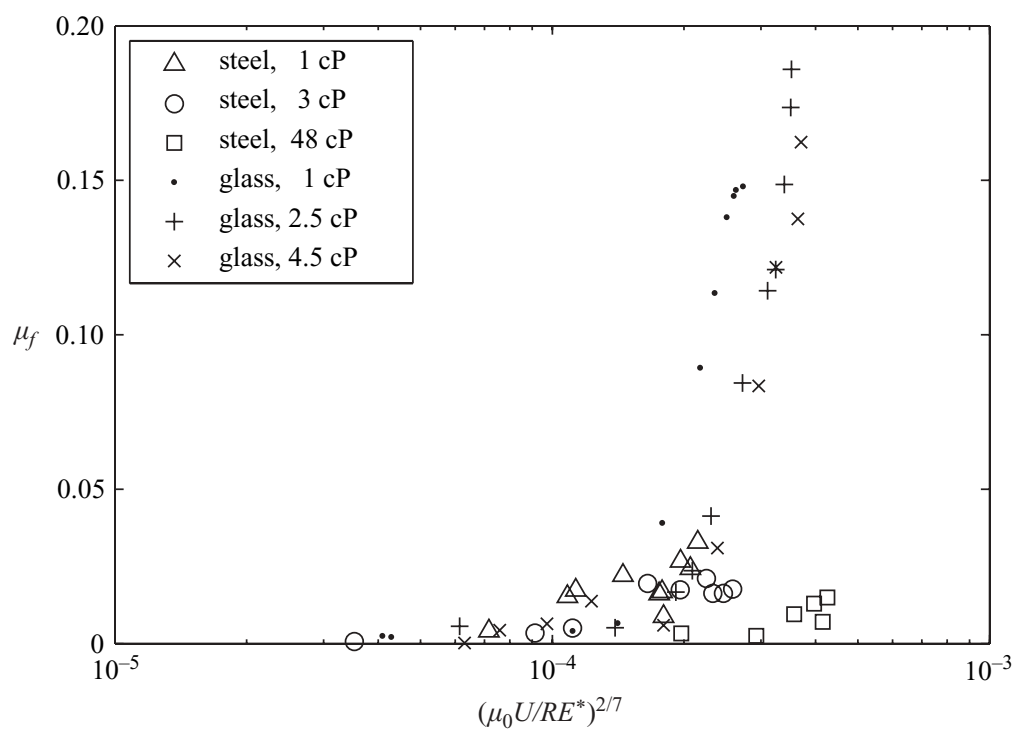

FiguRE 15. Coefficient of sliding friction, $\mu_{f}$, as a function of the shear strain of the system, $\left(\mu_{0} U / E^{*} R\right)^{2 / 7}$, for immersed oblique collisions in water and glycerol-water mixtures. Symbols $\triangle, \square, \bigcirc$ correspond to collisions with smooth particles and $\mathbf{Q},+, \times$ to collisions with rough particles.

Hence, $\mu_{f}$ can be represented as

$$
\mu_{f}=0.87\left(\frac{\mu_{0} U}{E^{*} R}\right)^{2 / 7}\left(\frac{1}{\eta E^{*}}\right)^{5 / 7}\left(\frac{\rho_{p} V^{2}}{E^{*}}\right)^{-6 / 35} A\left(\eta E^{*}, \rho_{p} V^{2} / E^{*}\right) .
$$

The first non-dimensional grouping, $\mu_{0} U / E^{*} R$, represents the shear strain of the system, and corresponds to the $U^{*}$ shear strain used by Gohar (2001); the second, $\eta E^{*}$, is a coupling coefficient between the solid and liquid phases; and the third one, $\rho_{p} V^{2} / E^{*}$, represents the normal strain of the system. The integral in (5.9) is a function $A=A\left(\eta E^{*}, \rho_{p} V^{2} / E^{*}\right)$ of the coupling coefficient and normal strain. For the conditions explored in this study, $\eta E^{*}$ is approximately 20 ; the product of the factors dependent upon the normal strain is nearly constant and equal to approximately 500 .

Figure 15 shows the experimental friction coefficients, from figures 8 and 11, as a function of the shear strain of the system, $\mu_{0} U / E^{*} R$. The friction coefficients for glass collisions where $\left(\mu_{0} U / E^{*} R\right)^{2 / 7}<10^{-4}$ collapse onto the same range as all steel collisions. At $\left(\mu_{0} U / E^{*} R\right)^{2 / 7} \approx 10^{-4}$, the collisions with glass spheres diverge from the group formed by the rest of the data and their friction coefficients rapidly climb toward a value similar to that of dry collisions. The observed trend for the friction coefficient in lubricated collisions is qualitatively consistent with EHD theory: as the shear strain of the system increases, the friction coefficient increases. In the cases with glass spheres, as the shear strain increases and the liquid in the gap hardens, a point is reached at which the surface roughness elements lock with the liquid in the gap (experimentally, $\left(\mu_{0} U / E^{*} R\right)^{2 / 7} \approx 10^{-4}$ ) causing the friction coefficient to jump to a value similar to that of dry systems. 


\section{Conclusion}

Measurements were obtained from immersed oblique collisions of rough glass particles and smooth steel particles with a smooth wall. Oblique collisions in a fluid were shown to exhibit characteristics similar to those of dry collisions. The results were described using a three-parameter model based on a normal coefficient of restitution, $e$, a rotational coefficient of restitution, $\beta$, and a coefficient of sliding friction, $\mu_{f}$. These parameters should prove useful for numerical simulations of multiphase flows. Currently, several simulation methods rely on lattice-based models that break down at small distances and that could benefit from an instantaneous collisional operator.

In immersed collisions, the rotational impulse experienced by rough particles may be a consequence of solid-solid contacts between the surface asperities of the particle and the wall. In cases with smooth particles, there is a substantial decrease in the rotational impulse when compared to collisions in air. In these cases, the lubrication effects result in a reduction in $\mu_{f}$ by almost an order of magnitude.

An analysis of the viscous shear in the lubrication gap for contacts of smooth spheres was performed. A model based on lubrication theory is proposed, which predicts, within experimental uncertainty, the frictional force experienced during the lubricated impact of a smooth sphere. The model depends on an assumed temperature increase within the lubrication layer, since the present set-up does not allow for the measurement of the temperature in the gap. Knowledge of the combined effects of temperature and pressure on the fluid viscosity is necessary in order to estimate the tangential force during impact. A numerical model that evaluates the temperature and pressure in the gap is currently difficult to attain.

We are grateful to P. C. Jennings for providing the Zerodur block used in the present study. We also thank C. E. Brennen and R. Zenit for the many fruitful discussions leading up to the preparation of this paper. Some assistance in the lab was provided by A. Ruiz-Angulo and F.-L. Yang. This work was supported by the National Science Foundation under grant CTS-9908430.

\section{Appendix. Torsional stiffness of the pendulum string}

Modelling the string as a linear torsional spring, the torsional oscillation of the pendulum is governed by the equation $I_{0} \theta^{\prime \prime}+K_{s} \theta=0$, where $I_{0}$ is the polar moment of inertia of the sphere about its centre and $K_{s}$ is the torsional stiffness of the string suspending it. The general solution is $\theta(t)=A \cos \omega_{0} t+B \sin \omega_{0} t$, where $\omega_{0}=\sqrt{K_{s} / I_{0}}=2 \pi f$. Therefore,

$$
K_{s}=4 \pi^{2} f^{2} I_{0}=\frac{32}{15} \pi^{3} \rho \frac{R^{5}}{\tau^{2}},
$$

where the polar moment of inertia of a solid sphere, $I_{0}=2 m R^{2} / 5=8 \pi \rho R^{5} / 15$, has been substituted in. By measuring the period $\tau$ of oscillations of a $12.7 \mathrm{~mm}$ steel ball-bearing $\left(\rho=7780 \mathrm{~kg} \mathrm{~m}^{-3}\right)$, the torsional stiffness of the different strings can be calculated from equation (A 1). The results are summarized in table 3.

The contact time for an elastic dry collision is of the order of $5 \mu$ s for contacts involving steel or glass. For collisions in which plastic deformation occurs, the period becomes longer. Zenit, Hunt \& Brennen (1997) showed that the duration of an immersed collision is somewhat protracted, presumably because the particle impact speed is reduced by the fluid. The amount of energy that can be recovered in the 


$\begin{array}{cccc}\text { String } & \varnothing(\mu \mathrm{m}) & \tau(\mathrm{s}) & K_{s} \times 10^{9}(\mathrm{~N} \mathrm{~m}) \\ \text { 6X } & 127 & 6.648 & 3846.4 \\ \text { 8X } & 76 & 17.229 & 572.8 \\ \text { 9X } & 51 & 41.568 & 98.4\end{array}$

TABLE 3. Torsional stiffness of pendulum strings.

tangential direction from an oblique contact is related to the lateral stiffness of the bodies in contact, $\kappa_{\text {contact }}$. The elastic time scales $\tau_{\text {collision }}$ and $\tau_{\text {string }}$, corresponding to the lateral stiffness of contact and the torsional stiffness of the string, respectively, are related by the ratio

$$
\frac{\tau_{\text {string }}}{\tau_{\text {collision }}}=\frac{\sqrt{\kappa_{\text {contact }} / m}}{\sqrt{K_{\text {string }} / I_{0}}} \approx \frac{1}{R} \sqrt{\frac{\kappa_{\text {contact }}}{K_{\text {string }}}},
$$

where $\kappa_{\text {contact }}=8 a G^{*}$ is the lateral stiffness of a sphere-plane contact. The reduced shear modulus, $G^{*}=\left[\left(2-v_{1}\right) / G_{1}+\left(2-v_{2}\right) / G_{2}\right]^{-1}$, depends on $G_{1}$ and $G_{2}$, the sphere and wall shear moduli, and $v_{1}$ and $v_{2}$, the respective Poisson ratios. Based on the times of contact for a typical steel-glass collision and on the measured periods of oscillation in table 3 , we have from equation (A 2 ) that $\kappa_{\text {contact }} / K_{\text {string }} \approx 10^{10}$. The torsional stiffness of the string can therefore be neglected when analysing oblique collisions with a pendular set-up like the one proposed. Furthermore, the stiffness of the string has no effect on the experimental measurement, since each experimental sample spans at most $0.2 \mathrm{~s}$, a duration two orders of magnitude shorter than the torsional stiffness timescale.

\section{REFERENCES}

Ånrström, B. O., Penchinat, C. \& Norrby, T. 2003 An experimental study of the influence of heat storage and transportability of different lubricants on friction under transient elastohydrodynamic conditions. Proc. Instn Mech. Engrs J: J. Engng Tribol. 217 (J1), 2737.

Avallone, E. A. \& Baumeister III, T. (ed.) 1987 Marks' Standard Handbook for Mechanical Engineers, 8th edn. McGraw-Hill.

BARNOCKY, G. \& DAVIS, R. H. 1988 Elastohydrodynamic collision and rebound of spheres: experimental verification. Phys. Fluids 31, 1324-1329.

BARNOCKY, G. \& DAVIS, R. H. 1989 The influence of pressure-dependent density and viscosity on the elastohydrodynamic collision and rebound of two spheres. J. Fluid Mech. 209, 501-519.

Bowden, F. P. \& TABOR, D. 1950 The Friction and Lubrication of Solids. Clarendon.

BRENNER, H. 1961 The slow motion of a sphere through a viscous fluid towards a plane surface. Chem. Engng Sci. 16, 242-251.

Cameron, A. 1981 Basic Lubrication Theory, 3rd edn. Ellis Horwood.

ten Cate, A., Nieuwstad, C. H., Derksen, J. J. \& Van den Akker, H. E. A. 2002 Particle imaging velocimetry experiments and lattice-Boltzmann simulations on a single sphere settling under gravity. Phys. Fluids 14, 4012-4025.

Cattaneo, C. 1938 Sul contatto di due corpi elastici: distribuzione locale degli sforzi. Accademia dei Lincei, Rendiconti, ser. 6, 21, 342-348, 434-436, 474-478.

Christensen, H. 1970 Elastohydrodynamic theory of spherical bodies in normal approach. Trans. ASME: J. Lubric. Tech. 92, 145-154.

Chu, P. S. Y. \& Cameron, A. 1962 Pressure viscosity characteristics of lubricating oils. J. Inst. Petrol. 48, 147-155. 
Davis, R. H., Rager, D. A. \& Good, B. T. 2002 Elastohydrodynamic rebound of spheres from coated surfaces. J. Fluid Mech. 468, 107-119.

Davis, R. H., Serayssol, J. M. \& Hinch, E. J. 1986 The elastohydrodynamic collision of two spheres. J. Fluid Mech. 163, 479-497.

Eisenberg, D. \& Kauzmann, W. 1969 The Structure and Properties of Water. Oxford University Press.

Elata, D. 1996 On the oblique compression of two elastic spheres. Trans. ASME: J. Appl. Mech. 63, 1039-1041.

Foerster, S. F., Louge, M. Y., Chang, A. H. \& Allia, K. 1994 Measurements of the collision properties of small spheres. Phys. Fluids 6, 1108-1115.

Glowinski, R., Pan, T. W., Hesla, T. I. \& Joseph, D. D. 1999 A distributed Lagrange multiplier fictitious domain method for particulate flows. Intl J. Multiphase Flow 25, 755-794.

GoHaR, R. 2001 Elastohydrodynamics, 2nd edn. Imperial College Press, London.

Goldsmith, W. 1960 Impact. Edward Arnold.

Gondret, P., Hallouin, E., Lance, M. \& Petit, L. 1999 Experiments on the motion of a solid sphere toward a wall: from viscous dissipation to elastohydrodynamic bouncing. Phys. Fluids 11, 2803-2805.

Gondret, P., Lance, M. \& Petit, L. 2002 Bouncing motion of spherical particles in fluids. Phys. Fluids 14, 643-652.

Hu, H. H. 1996 Direct simulation of flows of solid-liquid mixtures. Intl J. Multiphase Flow 22, 335-352.

Johnson, K. L. 1985 Contact Mechanics. Cambridge University Press.

Joseph, G. G., Zenit, R., Hunt, M. L. \& Rosenwinkel, A. M. 2001 Particle-wall collisions in a viscous fluid. J. Fluid Mech. 433, 329-346.

KantaK, A. A. \& Davis, R. H. 2004 Oblique collisions and rebound of spheres from a wetted surface. J. Fluid Mech. 509, 63-81.

Lun, C. K. K. \& SAVAGE, S. B. 1987 A simple kinetic theory for granular flow of rough, inelastic, spherical particles. Trans. ASME E: J. Appl. Mech. 54, 47-53.

Lundberg, J. \& Shen, H. H. 1992 Collisional restitution dependence on viscosity. J. Engng Mech. Div. ASCE 118, 979-989.

McLaughlin, M. H. 1968 An experimental study of particle-wall collision relating to flow of solid particles in a fluid. Thesis, California Institute of Technology, Pasadena, California.

Maw, N., Barber, J. R. \& Fawcett, J. N. 1976 The oblique impact of elastic spheres. Wear 38, 101-114.

Maw, N., Barber, J. R. \& FAWCEtT, J. N. 1981 The role of elastic tangential compliance in oblique impact. Trans. ASME F: J. Lubric. Tech. 103, 74-80.

Mindlin, R. D. 1949 Compliance of elastic bodies in contact. Trans. ASME: J. Appl. Mech. 16, $259-268$.

Mindlin, R. D. \& Deresiewicz, H. 1953 Elastic spheres in contact under varying oblique forces. Trans. ASME: J. Appl. Mech. 20, 327-344.

NGuYEn, N. \& LADD, A. J. C. 2002 Lubrication corrections for lattice-Boltzmann simulations of particle suspensions. Phys. Rev. E 66, 046708.

Potapov, A. V., Hunt, M. L. \& CAmpbell, C. S. 2001 Liquid-solid flows using smoothed particle hydrodynamics and the discrete element method. Powder Tech. 116, 204-213.

Smart, J. R. \& Leighton, D. T. 1989 Measurement of the hydrodynamic surface-roughness of noncolloidal spheres. Phys. Fluids A 1, 52-60.

Sundin, K. G. \& ÅHrström, B. O. 1999 Method for investigation of frictional properties at impact loading. J. Sound Vib. 222, 669-677.

Timoshenko, S. P. \& Goodier, J. N. 1970 Theory of Elasticity, 3rd edn. McGraw-Hill.

Walton, K. 1978 The oblique compression of two elastic spheres. J. Mech. Phys. Solids 26, 139150.

Walton, O. R. 1993 Numerical simulation of inelastic, frictional particle-particle interactions. In Particulate Two-Phase Flow (ed. M. C. Roco), chap. 25, pp. 884-911. ButterworthHeinemann.

Workel, M. F., Dowson, D., Ehret, P. \& TAYlor, C. M. 2001 Design and development of a ball impact apparatus for the direct measurement of lubricant friction under high pressures and shear rates. Proc. Instn Mech. Engrs J: J. Engng Tribol. 215 (J3), 211-222. 
Workel, M. F., Dowson, D., Ehret, P. \& Taylor, C. M. 2003 Measurements of the coefficients of friction of different lubricants during impact under high pressure and shear. Proc. Instn Mech. Engrs J: J. Engng Tribol. 217 (J2), 115-124.

Zenit, R., Hunt, M. L. \& Brennen, C. E. 1997 Collisional particle pressure measurements in solid-liquid flows. J. Fluid Mech. 353, 261-283.

Zhang, J. P., Fan, L. S., Zhu, C., Pfeffer, R. \& Qi, D. W. 1999 Dynamic behavior of collision of elastic spheres in viscous fluids. Powder Tech. 106, 98-109. 